\section{CASE REPORT}

C. Grommes

C. Griffin

K.A. Downes

A.J. Lerner

\title{
Steroid-Responsive Encephalopathy Associated with Autoimmune Thyroiditis Presenting with Diffusion MR Imaging Changes
}

\begin{abstract}
SUMMARY: Steroid-responsive encephalopathy associated with autoimmune thyroiditis (SREAT) presents with focal or diffuse nonenhancing MR imaging abnormalities in $50 \%$ of patients with SREAT during subacute exacerbation. Vasculitic changes in biopsy studies as well as the elevation of antithyroid antibodies and CSF protein suggests an inflammatory cause. We report the case of a patient with SREAT with changes on diffusion-weighted MR imaging, which improved with corticosteroid therapy and plasmapheresis, supporting the theory of inflammatory changes in exacerbation of presumptive SREAT.
\end{abstract}

$S_{\mathrm{d}}$ ince Brain et $\mathrm{al}^{1}$ first described a patient with Hashimoto disease and encephalopathy, more than 100 patients have been reported as having Hashimoto encephalopathy. ${ }^{2}$ It is now a well-recognized neurologic complication of autoimmune thyroid disease independent of thyroid status and is also called steroid-responsive encephalopathy with autoimmune thyroiditis (SREAT). ${ }^{2,3}$ Patients present with encephalopathy (100\%), seizures $(66 \%)$, myoclonus (38\%), neuropsychiatric symptoms (36\%), and strokelike symptoms (27\%). ${ }^{2}$ Patients are also found to have elevated thyroid antibodies, though the relationship between neurologic symptoms and signs and the type of thyroid disease as well as antibody concentration is unclear. ${ }^{2}$ Approximately $50 \%$ of patients with SREAT have nonenhancing MR imaging abnormalities with increased signal intensity on T2 and fast fluid-attenuated inversion recovery (FLAIR) imaging in the white matter as well as dural enhancement, ${ }^{4,5}$ which may abate with treatment. ${ }^{6}$ Results of biopsies from MR imaging changes in SREAT have demonstrated evidence of vasculitis. ${ }^{6}$ Diffusion-weighted MR imaging (DWI) is able to detect small, active, ischemic lesions not detected by conventional MR imaging, as seen in central nervous system (CNS) vasculitis, ${ }^{7,8}$ that may have a component of cytotoxic edema. We report the case of a patient with SREAT demonstrating abnormal results on DWI that abated after corticosteroid therapy and plasmapheresis. Therefore, the DWI changes in the case of our patient are also suggestive of an inflammatory vascular pathologic process as an underlying cause of SREAT.

\section{Case Report}

A 32-year-old right-handed woman presented with a 3-week history of confusion and psychosis. Insomnia had developed as well as visual and auditory hallucinations. Medical history included juvenile rheumatoid arthritis, untreated hypothyroidism, and idiopathic thrombocytopenic purpura (clinically inactive). She had fluctuating awareness suggestive of delirium or metabolic encephalopathy in addition to her acute psychosis. Results of CT scan of the head were unremark-

Received February 12, 2008; accepted after revision February 17

From the Departments of Neurology (C.G., C.G., A.J.L.) and Pathology (K.A.D.), University Hospitals Case Medical Center, Cleveland, Ohio.

Please address correspondence to Alan J. Lerner, Department of Neurology, University Hospitals Case Medical Center, 11100 Euclid Ave, Cleveland, $\mathrm{OH}$ 44106; e-mail: alan.lerner@UHhospitals.org

DOI 10.3174/ajnr.A1113 able. Abnormal laboratory results included thyroid-stimulating hormone (TSH) 17.93 mIUnits/L, antithyroid peroxidase (TPO) $>1000$ $\mathrm{IU} / \mathrm{mL}$, antithyroglobulin (TG) $1719 \mathrm{IU} / \mathrm{mL}$, antinuclear antibody (ANA) testing 1:320, platelet count 80,000-110,000, free T4 index 13.2 (normal up to 11), free T3 uptake 1.27 (normal up to 1.23 ). Results of CSF studies were normal except for a protein of $58 \mathrm{mg} / \mathrm{dL}$ and 2 oligoclonal bands. Results of additional serologic and CSF evaluations were negative.

On physical examination, vital signs and general medical examination were within normal limits. The patient was alert, cooperative, and oriented to person and time but had auditory hallucinations and illusions, loose associations and tangential thoughts, mild paranoid delusions, and no suicidal ideation. There was no speech or language deficit, and visuospatial constructions were normal. The Mini-Mental State Examination score was 30/30. Results of cranial nerve examination and the remainder of the neurologic examination were normal.

DWI demonstrated a punctate focus of increased signal intensity within the left centrum semiovale (Fig $1 A$ ) that was dark on apparent diffusion coefficient (ADC) mapping (Fig 1B). T2 and FLAIR images demonstrated areas of increased signal intensity corresponding to the areas of diffusion signal intensity abnormality in the hemispheric white matter (Fig $1 C,-D$ ). No abnormal enhancement was identified on the postcontrast $\mathrm{T} 1$ sequences.

The patient was diagnosed with SREAT and was treated with highdose intravenous corticosteroids followed by an oral taper. Her cognition improved dramatically, though mild confusion and mood disturbance continued. As the corticosteroids were tapered, psychosis recurred, and she was less responsive to a second course of high-dose corticosteroids. Plasmapheresis was initiated, with significant alleviation of the patient's symptoms. TSH was decreased to 0.58 after 60 days, anti-TPO to 55 after 60 days, and anti-TG to 158 after 60 days.

Results of follow-up MR imaging, done 30 days after initial treatment and before plasmapheresis, showed the punctate focus within the left centrum semiovale on DWI but without corresponding darkness on ADC mapping. The T2/FLAIR images again demonstrated areas of increased signal intensity abnormalities in the hemispheric white matter. Results of a second follow-up MR imaging examination, done 150 days after initial treatment and after plasmapheresis, showed no abnormal diffusion restriction, but there were still hemispheric white-matter changes on T2/FLAIR imaging.

\section{Discussion}

SREAT is a well-characterized clinical entity, though the relationship of anti-TPO and anti-TG antibodies to the pathogen- 

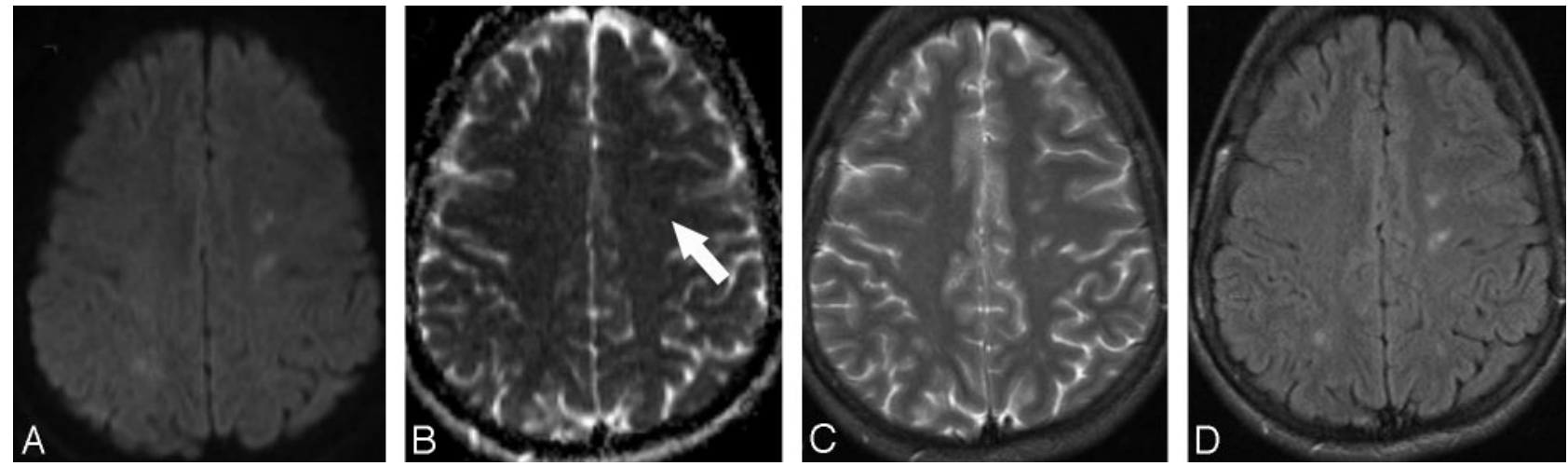

Fig 1. DWI $(A)$ initially demonstrates increased signal intensity within the left centrum semiovale with dark appearance on $A D C(B$; arrow). T2 $(C)$ and FLAIR $(D)$ images demonstrate areas of increased signal intensity corresponding to the areas of diffusion signal intensity abnormality in the white matter.

esis of the syndrome is unclear. ${ }^{2}$ Our patient would fulfill diagnostic criteria put forth in the largest clinical series to date. ${ }^{4}$ Bohnen et $\mathrm{al}^{9}$ first reported MR imaging changes in patients with SREAT, described as focal or diffuse nonenhancing MR imaging abnormalities on T2 or FLAIR sequences during subacute exacerbations of the disease. To our knowledge, this study is the first to demonstrate DWI changes in the setting of an acute exacerbation of SREAT and its improvement correlating with disease state and reduction in anti-TPO and anti-TG antibodies.

Although the pathogenesis of SREAT is not known at this time, elevated autoantibodies, elevated CSF protein, and histopathologic features of SREAT, including lymphocytic perivascular cuffs, ${ }^{2}$ and vasculitis of venules ${ }^{10,11}$ and arterioles ${ }^{11}$ suggest an inflammatory cause. Furthermore, previous reports ${ }^{6}$ have correlated MR imaging changes in SREAT with biopsy evidence of vasculitis. DWI is able to detect small, active, ischemic lesions not detected by conventional MR imaging. These DWI changes are also seen in CNS vasculitis and improve with treatment. ${ }^{7,8}$ As in our patient, immunosuppressive treatment led to an improvement in DWI and a reduction in autoantibody titers as well as improvement in clinical status, which suggests an inflammatory or vasculitic component in the genesis of SREAT. The initial DWI changes were associated with abnormal signal intensity on ADC mapping indicating an acute vascular pathologic process. With time, the DWI as well as the correlated ADC signal intensity improved.

The case of our patient underlines the importance to include DWI in the MR imaging evaluation of patients with SREAT to assess for possible vasculitic changes and provides another measure of response to treatment. Our patient, like others, did not display a consistent correlation of antibody titers with clinical symptoms. Plasmapheresis clearly lowered antibody titers acutely and was associated with alleviation in clinical symptoms. Although the cause of this syndrome remains obscure, it shares features with other causes of cerebral vasculitis, and overall prognosis remains good with several treatment modalities.

\section{References}

1. Brain L, Jellinek EH, Ball K. Hashimoto's disease and encephalopathy. Lancet 1966;2:512-14

2. Chong JY, Rowland LP, Utiger RD. Hashimoto encephalopathy: syndrome or myth? Arch Neurol 2003;60:164-71

3. Shaw PJ, Walls TJ, Newman PK, et al. Hashimoto's encephalopathy: a steroidresponsive disorder associated with high anti-thyroid antibody titers-report of 5 cases. Neurology 1991;41:228-33

4. Castillo P, Woodruff B, Caselli R, et al. Steroid-responsive encephalopathy associated with autoimmune thyroiditis. Arch Neurol 2006;63:197-202

5. Sawka AM, Fatourechi V, Boeve BF, et al. Rarity of encephalopathy associated with autoimmune thyroiditis: a case series from Mayo Clinic from 1950 to 1996. Thyroid 2003;13:227-28

6. Mahad DJ, Staugaitis S, Ruggieri P, et al. Steroid-responsive encephalopathy associated with autoimmune thyroiditis and primary CNS demyelination. J Neurol Sci 2005;228:3-5

7. White ML, Hadley WL, Zhang Y, et al. Analysis of central nervous system vasculitis with diffusion-weighted imaging and apparent diffusion coefficient mapping of the normal-appearing brain. AJNR Am J Neuroradiol 2007;28:933-37

8. Moritani T, Hiwatashi A, Shrier DA, et al. CNS vasculitis and vasculopathy: efficacy and usefulness of diffusion-weighted echoplanar MR imaging. Clin Imaging 2004;28:261-70

9. Bohnen N, Parnell K, Harper C. Reversible MRI findings in a patient with Hashimoto's encephalopathy. Neurology 1997;49:246-47

10. Shibata N, Yamamoto Y, Sunami N, et al. [Isolated angiitis of the CNS associated with Hashimoto's disease]. Rinsho Shinkeigaku 1992;32:191-98

11. Nolte KW, Unbehaun A, Sieker H, et al. Hashimoto encephalopathy: a brainstem vasculitis? Neurology 2000;54:769-70 